\title{
ITO islands as floating electrodes to deposit aligned carbon nanotubes for photovoltaic applications
}

\begin{abstract}
Recently transparent conductive electrodes (TCEs) have attracted the interest of researchers due to their outstanding optical and electrical properties. Indium tin oxide (ITO) is an oxide material that combines the criteria of high conductance and transmittance. However, ITO is a non-flexible, brittle and expensive material. TCEs are commonly embedded in solar cells to perform high photon absorption and electron collection instantaneously. In this paper, an optimized TCE network is proposed to maintain TCEs' conductivity and transparency and to improve their flexibility and ability to handle mechanical forces. The network is designed by aligning multi-walled carbon nanotubes (MWCNTs) on ITO speckled surface to form ITOCNT grid. The alignment mechanism is achieved through dielectrophoretic (DEP) force, where an electric field of $10 \mathrm{~V}$ and $105 \mathrm{~Hz}$ is subjected to an ethanol/ MWCNT suspension to assemble the CNT across ITO's floating electrodes.
\end{abstract}

Keyword: MWCNT; TCEs; Alignment; DEP; Thin film solar cell 